\title{
Stretching Shielding Calculations
}

\section{A. Van Ginneken}

June 1979

\section{Abstract}

A scaling rule is presented for use in solving hadron shielding problems when the answer for geometrically related configurations is known. Extensions to non-hadron shielding are indicated.

\section{Introduction}

When shielding decisions must be made on very short notice, an educated guess based on available materia ${ }^{1}$ often becomes necessary. This note concerns a simple guiding rule which may be helpful for this purpose. In certain instances this rule is nearly exact and therefore useful even when the design of a shield proceeds at a more leisurely pace. For most applications the approximations involved will necessarily be rather crude and will not offer an effective alternative to specific calculations. The emphasis of this note is on estimating hadron dose rates. other applications are briefly discussed.

The physical bases of the rule are: (1) in the highenergy region ( $\geq 10 \mathrm{GeV}$ ) the characteristics of particlenucleus interactions are not very sensitive to nuclear size, (2) shielding calcuations combine effects of many generations of the internuclear cascade which tends to further reduce nuclear size dependence, (3) above 50-100 MeV the collision 
length of the cascading particles is roughly independent of energy and (4) elastic scattering and ionization losses are of lesser importance than particle production in determining the general characteristics of high energy hadron cascades.

\section{Homogeneous Targets}

The first principle involved is most easily seen by considering the archetypical geometry of CASIM $^{2}$ as well as other hadron cascade codes, i.e. that of a very large homogeneous cylinder into which a pencil beam of energetic protons is introduced. Assume that such a calculation has been performed for a given material and that it is now desired to study an identical situation for the. same material but in this case having only half the density (e.g. liquid vs solid phase). The beam energy must be essentially the same in both cases. Because the collision length varies inversely with density all distances in the second case are twice that of the first and since the collision length is the only parameter which differs between the calculations it follows that:

$$
S_{2}(x, y, x)=(1 / 8) S_{1}(x / 2, y / 2, x / 2)
$$

where $S_{1}, S_{2}$ denote star densities for the heavier and lighter materia 1 and the factor (1/8) comes about because space has three dimensions.

The quantity usually of most interest is the dose rate. In regions where the cascade is well developed dose rate is proportional to flux or number of particles per unit area, $\phi$, incident on the human body or on a detector: 


$$
D_{i}(x, y, z)=K \phi_{i}(x, y, z) \quad(i=1,2)
$$

The flux, in turn, is related to star density via the collision length, $\lambda_{i}$, of the shielding material

$$
\phi_{i}(x, y, z)=\lambda_{i} S_{i}(x, y, z) \quad(i=1,2)
$$

In the previous example $\lambda_{2}=2 \lambda_{1}$, and it follows that

$$
D_{2}(x, y, z)=(1 / 4) D_{i}(x / 2, y / 2, z / 2)
$$

Note that except for some reservations about eq. (2) everything is exact even if the example is somewhat contrived.

There is some practical use for the above in deriving results for soil from calculations for concrete and vice versa. Since their composition is nearly identical the "stretching factor" is the ratio of their densities, i.e. about 1.2. Thus

$$
D_{c}(x, y, z)=(1 / 1.44) D_{c}\left(x_{c} / 1.2, y_{c} / 1.2, z_{c} / 1.2\right)
$$

where $D_{S}, D_{C}$ are dose rates in homogeneous soil and concrete respectively.

If one deals with two materials of differing composition then eq. (4) generalizes to

$$
D_{2}(x, y, z)=\left(\lambda_{1} / \lambda_{2}\right)^{2} D_{1}\left(\lambda_{1} x / \lambda_{2}, \lambda_{1} y / \lambda_{2}, \lambda_{1} z / \lambda_{2}\right)
$$

It is obvious that the $\lambda_{i}$ refer to distance and not to mass per unit area. This is the case throughout this note. Since the $\lambda_{i}$ will always appear as quotients either collision lengths (including elastic processes) or absorpotion lengths may be used, given the approximate nature of the procedure. 


\section{More Complicated Geometries}

Another typical CASIM geometry is that of a targetin-a-cave. Assume it is desired, while holding target material as well as target and inside cave dimensions constant, to study the effects of substituting different materials in the walls of the cave. Clearly the uniform stretching arguments no longer apply. Some insight is provided by drawing rays representing extended trajectories of particles produced in the target. Except for backscattering into the cave one can argue as before since uniform stretching holds inside the walls. This is illustrated in Fig. 1 using again a stretching factor of two. However, the rule is to be applied ray by ray and the information needed for this is not available in Ref. 1 or in a regular CASIM run. For any given location in the stretched version it is possible to derive an approximate star density only if for all rays which contribute significantly application of eq. (6) involves roughly the same location in the reference calculation. The success may depend strongly on position as illustrated in Fig. 1 with the help of a few selected rays. It is clear that one would likely succeed better in estimating the dose rate at location $E$ than at $A$. For a more quantitative treatment one needs to take into consideration the contribution of each ray was well as the density of rays, i.e. energy and angular distribution of the particles produced in the target. However, to arrive at a definite algorithm it is perhaps better to be guided by a yet more general (and realistic) geometry. The present example will emerge as a special case. 
Assume a proton beam strikes a beryllium target placed inside a magnet string surrounded by a cave with iron walls. Again, assume that such a case has been calculated and one wishes to estimate the effects of changing the walls to concrete. The situation suitably idealized and cast into cylindrically symmetric form is shown in Fig. 2. Specifically one seeks the dose rate at location A for the concrete case.

\section{Homogenizing and Stretching}

To introduce the second principle involved in the algorithm an "effective" collision length is introduced. This may be defined as the distance along a cascade trajectory between the point where the beam enters and location A divided by the number of collisions and suitably averaged over all such paths. For simplicity, instead of averaging over all trajectories the effective collision length is conveniently evaluated along one representative trajectory. In Fig. 2 some possible trajectories are shown. The one labelled $\varepsilon$ displays the typical random walk character of a cascade trajectory. The others, because they suffer only a few well placed interactions and because of the fortuitous character of the production angles, represent more stylized versions. These are however much easier to deal with geometrically. The idealization is extended to the radial direction where the entire trajectory projects onto a radius vector. If one were to pick a "typical" trajectory from among the stylized ones the path labelled a would be excluded immediately. The others are more likely to occur but for sake of definiteness let the path $\gamma$ be selected. The effective collision length is then 


$$
\Lambda(x, y, z)=s_{\gamma} d s /\left(s_{\gamma} d s / \lambda(x, y, z)\right)
$$

where $\delta_{\gamma}$ denotes the line integral along $\gamma$ which is taken with respect to distance, $s$, along the trajectory. Note that $\Lambda$, in contrast with $\lambda$, is a continuous function of location in the geometry. This function combines certain properties of the geometry, material composition and cascade development.

In addition a direction-dependent effective collision length is introduced

$$
\Lambda_{x}(x, y, z)=\int_{\gamma} d x /\left(\int_{\gamma} d x / \lambda(x, y, z)\right)
$$

where the 1 ine integrals are now with respect to the $x$-axis. There are similar expressions for $\Lambda_{y}, \Lambda_{z}$.

For a stylized trajectory the 7 ine integrals appearing in eqs. (7) and (8) reduce to simple, easily evaluated algebraic expressions.

For the purpose of formulating an algorithm the effective collision lengths may be viewed as a means to "homogenize" the geometry, albeit in anisotropic fashion. The assumption is made that the flux at any given point $(x, y, z)$ in a given geometry equals the flux at the same location $(x, y, z)$ in a homogenized shield characterized by constant effective collision lengths $\Lambda_{x}, \Lambda_{y}, \Lambda_{z}, \Lambda$ as given by eqs. (7) and ( 8 ). In symbols

$$
\Phi(x, y, z)=\phi(x, y, z)
$$

where $\Phi, \phi$ denote the fluxes outside the homogenized shield and problem geometry, respectively. Eq. (9) translates into a relation between star densities

$$
S(x, y, z)=(\Lambda / \lambda) \Sigma(x, y, z)
$$


where $\Sigma$ is the star density in the homogenized shield and, as before, $\lambda$ is the collision length of the material at $(x, y, z)$ in the problem geometry (i.e. concrete, in the example). There exists a set of relations corresponding to eqs. (7) - (11) which relate collision length, flux and star density of the reference geometry and its homogenized version.

The connection between homogenized geometries is now readily made by introducing stretching factors, $\tau_{i}$, much 1 ike the ones which connect homogeneous targets. The basic difference is that for the general case the stretching factors are no longer isotropic

$$
\tau_{x}=\Lambda_{x} / \Lambda_{R, x}
$$

where $\Lambda_{R, x}$ is the effective collision length of the reference geometry with respect to $x$, taken along a trajectory corresponding to that of the problem geometry. There are similar definitions for $\tau_{y}, \tau_{z}$. For brevity, the dependence on location is no longer explitely shown in eq. (11) and in most of the seque1. The stretching factors define a oneto-one correspondence between problem and reference geometry, e.g.

$$
x_{R}=\tau_{x}^{-1} x
$$

and also appear in the Jacobian relating star densities of the homogenized geometries:

$$
\Sigma(x, y, z)=\tau_{x}^{-1} \tau_{y}^{-1} \tau_{z}^{-1} \Sigma_{R}\left(\tau_{x}^{-1} x, \tau_{y}^{-1} y, \tau z^{-1} z\right)
$$




\section{Algorithm}

The basic formulation of the algorithm can be presented diagramatically as follows:

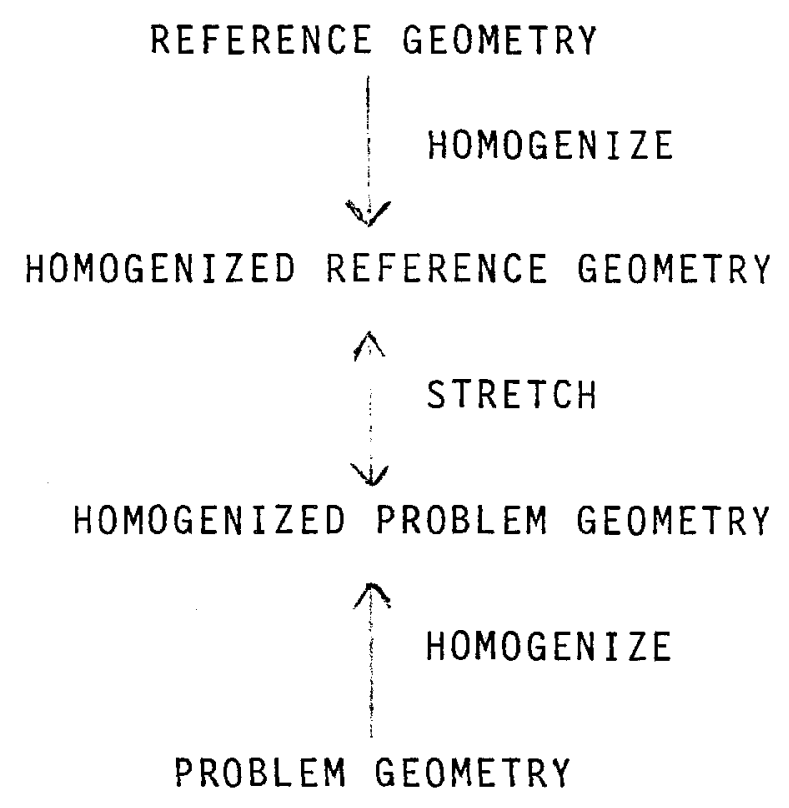

The rules for the three steps involved are given by eqs. (9) and (13). By substitution the basic expressions of the algorithm are obtained

$$
S(x, y, z)=\left(\Lambda / \Lambda_{R}\right)\left(\lambda_{R} / \lambda\right) \tau_{x}^{-1} \tau_{y}^{-1} \tau_{z}^{-1} S_{R}\left(\tau_{x}^{-1} x, \tau y^{-1} y, \tau z^{-1} z\right)
$$

from which

$$
D(x, y, z)=\left(\Lambda / \Lambda_{R}\right) \tau_{x}^{-1} \tau_{y}^{-1} \tau_{z}^{-1} D_{R}\left(\tau_{x}^{-1} x, \tau_{y}^{-1} y, \tau z^{-1} z\right)
$$


Note that the uncertainty in picking a typical trajectory enters only through ratios of effective collision lengths. This justifies the convenience of a stylized trajectory.

It is perhaps worthwhile to apply this explicitely to the example of Fig. 2. For the radial coordinate eqs. (11) and (12) lead to

$$
\tau_{r}=r / r_{R}=\Lambda_{r} / \Lambda_{R, r}
$$

with reference to Fig. 2 and the $\gamma$ trajectory.

$$
\left.\Lambda_{r}(r, z)=r r_{1} / \lambda_{B e}+\left(r_{3}-r_{2}\right) / \lambda_{M}+\left(r-r_{4}\right) / \lambda\right]^{-1}
$$

where $\Lambda_{r}=\Lambda_{x}=\Lambda_{y}$, because of symmetry and $\lambda_{M}$ is an averaged collision length for the magnet materials. There is a similar expression for $\Lambda_{z}$.

Likewise,

$$
\Lambda_{R, r}\left(r_{R}, z_{R}\right)=r_{R}\left[r_{1} / \lambda_{B e}+\left(r_{3}-r_{2}\right) / \lambda_{M}+\left(r_{R}-r_{4}\right) / \lambda_{R}{ }^{-1}\right.
$$

and it follows from eqs. (16) that

$$
\left(r_{R}-r_{4}\right) / \lambda_{R}=\left(r-r_{4}\right) / \lambda
$$

and

$$
\left(z_{R}-z_{4}\right) / \lambda_{R}=\left(z-z_{4}\right) / \lambda
$$

By application of eqs. (19) and (20) it is easily shown that

$$
\Lambda / \Lambda_{\mathrm{R}}=\mathrm{s} / \mathrm{s}_{\mathrm{R}}
$$


where $s, s_{R}$ are the total length of the typical trajectory in problem and reference geometry, respectively.

In this example, $r_{R}$ is readily found from eq. (19) since $r_{4}$ is the tunnel radius, independent of the choice of typical trajectory. The value of $z_{4}$, on the other hand, varies with this choice and it (or $z_{R}$ directly) must be evaluated e.g. by graphical means. The same is true for $s$ and $s_{R}$. The final result is then

$$
D(r, z)=\left(s / s_{R}\right)\left(r_{R} / r\right)^{2}\left(z_{R} / z\right) D_{R}\left(r_{R}, z_{R}\right)
$$

This equation holds generally, with obvious extension to the non-cylindrically symmetric case. The same is not true for eqs. (17) - (20) which relate $\left(r_{R}, z_{R}\right)$ to $(r, z)$.

It is obvious that results will be more reliable the closer problem and reference geometry are related. No scaling should be attempted between geometries so disparate as to make evaluation of the stretching factors questionable.

It is clear that the procedure hinges strongly on the existence of a rather unambiguous typical trajectory and on one's ability to identify it. Where two or more such pathways are thought to compete the procedure would become at best more cumbersome. It is also clear that, since the typical trajectory will depend upon location, in those instances where eq. (22) must be evaluated many times the burden of this will quickly outpace that of recoding the geometry routine in CASIM. It is, however, possible to avoid computational labor by coding eq. (22) into CASIM and applying it to the results at the end of the Monte Carlo stage of the program. 


\section{Test Cases}

Sufficient experience with the algorithm needed to make general, quantitative statements about its reliability is lacking. This would be a difficult matter in any case in view of the diversity of applications, separation of the statistical component of the discrepancies, uncertainty of the representative trajectory, etc. Three tests have been run which offer a good indication as to the validity of the algorithm.

The first case is the example of Fig. 2 discussed at some length, above. The beryllium target is $40 \mathrm{~cm}$ long and $4 \mathrm{~cm}$ in radial extent. The magnet string is assumed to be of iron with $10 \mathrm{~cm}$ inner radius and $40 \mathrm{~cm}$ outer radius. The cave has an inner radius of $100 \mathrm{~cm}$ and outer radius of $200 \mathrm{~cm}$. The total length of the configuration is $1000 \mathrm{~cm}$. Two calculations were performed one using iron (reference) and the other concrete (problem) as cave wall materials. To minimize statistical fluctuations the calculations were run with correlated random numbers ${ }^{3}$. The ratios of results obtained from the calculation for the iron shield via scaling to those computed directly for concrete are shown in Table I at four depths and three radii. The results are quite close particularly since a large portion of the discrepancy is likely of a non-scaling origin. The average of all twelve predicted to observed star densities is 0.99 . The standard deviation of the distribution of the ratios is 0.21 . The principal source of error is expected from the fact that the finite volume bins which serve to estimate star density do not conform to the scaling rule, either in size or location, as would be desirable for this test. The results of Table I are based on interpolation between four neighboring bins 
both for iron and concrete. The random inter-bin variations encountered in each case are at least as large as the discrepancies of the scaling test shown in Table I.

The second case relates a multimedia geometry to a homogeneous target used as reference. The example of Fig. 2, slightly modified, serves again as the problem geometry. The only difference is that the target has been extended the full length of the configuration. Otherwise the scaling from a homogeneous target could not be readily made. The representative trajectory resembles the $\gamma$-trajectory of Fig. 2 except that the initial leg now extends $42.5 \mathrm{~cm}$ $\left(=\lambda_{B e}\right.$ ) instead of $20 \mathrm{~cm}$ (= half the target length). The rest of the procedure is the same as before. Results are shown in Table II. Not surprisingly the agreement here is poorer than for the first case. This is not only because scaling is less valid here but also because the use of correlated random number is less effective. The average of the set of ratios of Table II is 1.26 and the standard deviation is 0.56 . For many applications this is still acceptable.

The third case is entirely similar to the second except that the reference geometry is a homogeneous iron cylinder. Results are shown in Table III. They are comparable to the second case. The average and standard deviation of the ratios is 1.22 and 0.66 , respectively.

Comparing Tables II and II one learns in a roundabout way something about scaling between homogeneous concrete and iron targets. From the strong correlation between corresponding entries in the tables it is clear that the scaling between the two homogeneous targets holds better than between disparate geometries (though this also reflects the differences in effectiveness of correlated random numbers). 
Overal1, the comparisons, though 1 imited in scope, are quite gratifying. Aside from the expected correlations between Tables II and III there is no general trend discernible. A test of the sensitivity of these results to various choices of trajectories has not been attempted at this time.

\section{Other Applications}

The algorithm presented in this note has been explicitely formulated to calculate star densities and dose rates. There are obvious extensions to such problems as activation, detector response, etc. In general the rule cannot be applied to estimate energy deposition densities in hadron showers. Because of the electromagnetic component, such a calculation involves both the collision length and radiation length while the present procedure rests on the existence of a single scaling parameter. For the same reason care must be taken in estimating dose rates in regions where the electromagnetic component is important. However, the algorithm is valid for relating targets of identical composition but of different densities. Presumably scaling between materials close in atomic number will still yield fairly reliable results.

The scaling rule could be applied to pure electromagnetic cascades using the radiation length (and effective radiation length, etc.) in lieu of the collision length. The rule would roughly amount to application of the so-called Approximation $A$, which neglects ionization losses of the shower electrons ${ }^{4}$. In general the rule would be of a very approximate character. In muon shielding collision losses are virtually always a dominant mode of energy transfer. This appears to rule out scaling rules of this type.

I wish to thank M. Awschalom and L. Coulson for their comments. 


\section{References}

1. A. Van Ginneken and M. Awschalom, "High Energy Particle Interactions in Large Targets. Vol. 1", FNAL, Batavia, IL (1974). Or it may be based on actual experience in similar configurations.

2. A. Van Ginneken, "CASIM. A Program to Simulate Hadronic Cascades in Bulk Matter", FN-272, FNAL, Batavia, IL, Jan. 1975 .

3. In both cases a three-1ayered hierarchy of random numbers is used. This insures not only that the cascade development in the target and magnet string is identical but that it will be quite similar in the iron/concrete cave wall as well because they derive from identical strings of random numbers, hence random variables.

4. B. Rossi, "High Energy Particles", Prentice Hall, Englewood Cliffs, N. J., 1952. 


\section{Table Captions}

Table I. Ratio of star densities obtained by using the scaling rule and by direct calculation. The geometry of Fig. 2 is used with iron cave walls in the reference geometry and concrete in the problem geometry. The discrepancy is expected to contain a large component of nonscaling origin.

Table II. Ratio of star densities obtained by using the scaling rule and by direct calculation. The geometry of Fig. 2 with the target length extending over the entire configuration is the problem geometry. The reference geometry is a homogeneous concrete cylinder. The discrepancy is expected to contain a large component of non-scaling origin.

Table III. Ratio of star densities obtained by using the scaling rule and by direct calculation. The long-target geometry of Fig. 2 is the problem geometry. The reference geometry is a homogeneous iron cylinder. The discrepancy is expected to contain a large component of non-scaling origin. 
Table I

\begin{tabular}{|c|c|c|c|c|}
\hline $\mathrm{r}, \mathrm{cm} \quad \mathrm{z}, \mathrm{cm}$ & 100 & 400 & 700 & 980 \\
\hline 120 & 0.88 & 0.77 & 1.00 & 1.37 \\
\hline 160 & 1.27 & 1.01 & 1.16 & 1.10 \\
\hline 196 & 0.97 & 0.73 & 0.72 & 0.88 \\
\hline
\end{tabular}

Table II

\begin{tabular}{|c||c|c|c|c|}
\hline$r, \mathrm{~cm}$ & & & & \\
\hline 120 & 100 & 400 & 700 & 980 \\
\hline 160 & 0.72 & 1.09 & 2.43 & 1.04 \\
\hline 196 & 1.00 & 1.15 & 1.47 & 1.05 \\
\hline & 0.60 & 0.91 & 1.47 & 2.20 \\
\hline
\end{tabular}

Table III

\begin{tabular}{|c||l|l|l|l|}
\hline$r, \mathrm{~cm}$ & & & & \\
\hline 120 & 100 & 400 & 700 & 980 \\
\hline 160 & 0.67 & 1.12 & 1.73 & 0.75 \\
\hline 196 & 0.85 & 1.04 & 1.48 & 0.78 \\
\hline
\end{tabular}




\section{Figure Captions}

Fig. 1 Target-in-a-cave geometry where only the material in the walls is being changed. The homogeneous scaling rules are applied inside the cave walls for various rays. It is expected that star densities vary more strongly among locations $B, C$, and $D$ than among $F, G$, and $H$. This would make it more difficult to estimate the star density at $A$ in the stretched version than at $E$.

Fig. 2 Beam line geometry with beam striking a target located in a magnet string placed in a cave.

The change in material of the walls is studied. The paths $\alpha-\varepsilon$ represent trajectories of the internuclear cascade which contribute to the star density at location $A$. 